\title{
Niche Differentiation in the Rhizosphere and Endosphere Fungal Microbiome of Wild Paris polyphylla Sm.
}

\author{
Yan Wang ${ }^{\text {Corresp., } 1,2,3}$, Hanping Wang ${ }^{4}$, HuYin Cheng ${ }^{5}$, Fan Chang ${ }^{1,2}$, Yi Wan ${ }^{2,3}$, Xiaoping She ${ }^{\text {Corresp. } 1}$ \\ ${ }^{1}$ College of Life Sciences, Shaanxi Normal University, xi'an, China \\ 2 Shaanxi Microbiology Institute, xi'an, China \\ 3 Shaanxi Academy of Sciences, Engineering Center of QinLing Mountains Natural Products, Xi'an, Shaanxi, China \\ 4 College of Medical, Xi'an International University, xi'an, china \\ 5 College of Pharmacy, Shaanxi University of Chinese Medicine, xi'an, Shaanxi, China \\ Corresponding Authors: Yan Wang, Xiaoping She \\ Email address: wangyan-2012@foxmail.com, correspond2020@qq.com
}

Background. The plant microbiome is one of the key determinants of plant health and metabolite production. The plant microbiome affects the plant's absorption of nutrient elements, improves plant tolerance to negative environmental factors, increases the accumulation of active components, and alters tissue texture. The microbial community is also important for the accumulation of secondary metabolites by plants. However, there are few studies on the niche differentiation of endophytic microorganisms of plants, especially at different elevations.

Methods. We investigated the effects of altitude on the community composition of endophytic fungal communities and the differentiation of endophytic microorganisms among different niches in Paris polyphylla Sm. The rhizosphere soil, roots, rhizomes and leaves of wild-type Paris polyphylla Sm. at different altitudes were sampled, and the fungal communities of all samples were analysed by ITS 1 amplification sequencing.

Results. The results showed that in rhizosphere soil, the number of operational taxonomic units (OTUs) that could be classified or identified decreased significantly with increasing altitude, whereas in the endosphere of plants, the total number of OTUs was higher for the intermediate altitudes than for the other altitudes. Furthermore, the structural variability in the rhizosphere fungal community was significantly lower than that in the endophytic communities. In addition, our results confirmed the presence of niche differentiation among members of the endophytic microbial community. Finally, we also determined that the predominant genus of mycobiota in the rhizome was Cadophora. This study provides insight into the relationships between the endosphere microbiome and plants and can guide the artificial cultivation of this plant. 
1 Niche Differentiation in the Rhizosphere and Endosphere Fungal Microbiome of

2 Wild Paris polyphylla Sm.

3 Yan Wang ${ }^{1,2,3}$, HanPing Wang ${ }^{4}$, HuYin Cheng ${ }^{5}$, Fan Chang ${ }^{1,2}$, Yi Wan ${ }^{2,3}$, XiaoPing She ${ }^{1 *}$

$4 \quad{ }^{1}$ College of Life Sciences, Shaanxi Normal University, Xi'an, Shaanxi, People's Republic of

5 China

$6 \quad$ 'Shaanxi Microbiology Institute, Xi'an, Shaanxi, People's Republic of China

7 3Shaanxi Academy of Sciences, Engineering Center of QinLing Mountains Natural Products,

8 Xi'an, Shaanxi, People's Republic of China

$9{ }^{4}$ College of Medical, Xi'an International University, Xi'an Shaanxi, People's Republic of China

$10{ }^{5}$ College of Pharmacy, Shaanxi University of Chinese Medicine, Xi Xian New Area, People's

11 Republic of China

13 Corresponding Author:

14 XiaoPing She ${ }^{1}$

15 College of Life Sciences, Shaanxi Normal University, Xi'an, ShaanXi,

16 Email address: correspond2020@qq.com

17 Abstract

Background. The plant microbiome is one of the key determinants of plant health and metabolite production. The plant microbiome affects the plant's absorption of nutrient elements, improves plant tolerance to negative environmental factors, increases the accumulation of active components, and alters tissue texture. The microbial community is also important for the accumulation of secondary metabolites by plants. However, there are few studies on the niche differentiation of endophytic microorganisms of plants, especially at different elevations.

Methods. We investigated the effects of altitude on the community composition of endophytic fungal communities and the differentiation of endophytic microorganisms among different niches in Paris polyphylla Sm. The rhizosphere soil, roots, rhizomes and leaves of wild-type Paris polyphylla $\mathrm{Sm}$. at different altitudes were sampled, and the fungal communities of all samples were analysed by internal transcribed spacer (ITS) 1 amplification sequencing.

Results. The results showed that in rhizosphere soil, the number of operational taxonomic units (OTUs) that could be classified or identified decreased significantly with increasing altitude, whereas in the endosphere of plants, the total number of OTUs was higher at intermediate altitudes than other altitudes. Furthermore, the structural variability in the rhizosphere fungal community was significantly lower than that in the endophytic communities. In addition, our results confirmed the presence of niche differentiation among members of the endophytic microbial community. 
Cadophora. This study provides insight into the relationships between the endosphere microbiome and plants and can guide the artificial cultivation of this plant.

\section{Introduction}

Interactions between microorganisms and plants have become a popular research topic in microbiology and botany, with studies conducted on tree (Beckers et al., 2017; Cregger et al., 2018), crop and floriculture microbiomes. Plants and microorganisms have close mutualistic or competitive relationships. In most cases, microorganisms play key roles in the survival and performance of plants (Hackstein et al., 2010; Hacquard et al., 2015). In addition, microorganisms are also important for the regulation of plant immune systems (Jones et al., 2006; Khau et al., 2011; Lebeis et al., 2015; Lee et al., 2010) and influence plant metabolism (Khan et al., 2011; Khan et al., 2011). Microorganisms can influence medicinal plants in different ways, such as by affecting the absorption of nutrient elements, improving tolerance to stress, increasing the accumulation of plant active components, and causing changes in tissue texture. The presence of microorganisms in plants is also affected by the host plant genotype (Cregger et al., 2018). To some extent, microorganisms are specific to their hosts. Even within the same genus, endosphere microbiome communities may vary significantly.

The symbiosis between fungi and medicinal plants involves a series of complex processes, such as cell morphological changes, signal recognition, signal transduction, nutrient exchange and gene expression (Huang et al., 2018). Endophytic fungi produce the same secondary metabolites as host plants (Guo 2016; Lu et al., 2018; Huang et al., 2007; Xue et al., 2013). Some endophytic fungi can directly or indirectly affect the secondary metabolism of medicinal plants (Yadav et al., 2013; Bao et al., 2017). In addition, the associated bacterial communities may play important roles in the regulation of the plant immune system (Kau et al., 2011; Lebeis et al., 2015). Therefore, endosphere microbiome communities are often called the second or extended genome of the host (Beckers et al., 2017). Recently, the effects of endosphere fungi on the growth, development, environmental stress resistance and secondary metabolite synthesis of medicinal plants have attracted increased concern.

Plant - Microbe interactions has received substantial attention in recent years as a subject of scientific and commercial interest (Turner et al., 2013).Studies have shown that the content of secondary metabolites from the same medicinal plant species can be different depending on their location of cultivation, which could in part be related to different composition in their associated microbes when grown at different sites (Huang et al., 2018; Köberl et al., 2013). For some microbes, their metabolites could also be involved in modulating the production of bioactive phytometabolites, such as paclitaxel produced by Taxomyces andreanae (Köberl et al., 2013).

Paris polyphylla $\mathrm{Sm}$. is a famous medicinal plant belonging to Paris in the Liliaceae family. This plant is found in the tropical and temperate regions of Eurasia and is mainly distributed in Southwest China around altitudes of $900-2000 \mathrm{~m}$. The rhizome is the medicinal part of the plant. Plant components reach the required effectiveness after 5 years. The chemical components of this plant have haemostatic and anti-inflammatory properties as well as good therapeutic effects in 
treating snake bites. In China, Paris polyphylla $\mathrm{Sm}$. is used as a raw material for many medicines. There are many studies on the chemical composition and application depth of this plant, but the endosphere microbiome is less understood. However, wild Paris polyphylla Sm. is very rare. This study focuses on the endosphere microbiome of wild Paris polyphylla $\mathrm{Sm}$. The fungal communities of all samples were analysed by internal transcribed spacer (ITS) 1 amplification sequencing. We assessed the fungal microbiomes in rhizosphere soil, rhizomes, and leaves of Paris polyphylla Sm. at different altitudes; DNA samples were amplified by PCR and analysed by sequencing. The niche differentiation of the related fungal microbiome was also investigated. These studies will help us understand the correlation between the endosphere microbiome and plants and help guide the artificial cultivation of plants.

\section{Materials \& Methods}

\section{Field trials and sampling}

The samples were collected from the southern slope of Qinling Mountains, Han Zhong, Shaanxi Province, China. The location was at a longitude of $107^{\circ} 41^{\prime} \sim 107^{\circ} 50^{\prime} \mathrm{E}$ and latitude of $32^{\circ} 16^{\prime} \sim$ $32^{\circ} 43^{\prime} \mathrm{N}$. All samples were healthy and free of pests and diseases. All of the sampled trees were 4-5 years old based on bud scale scars. The sampling was carried out on plants with heights of 40$70 \mathrm{~cm}$ and on 8-11 leaves per plant. Sampling was carried out at three different altitudes, $1200 \pm 50$ $\mathrm{m}, 1500 \pm 50 \mathrm{~m}$, and $1800 \pm 50 \mathrm{~m}$, with 3 plants at each altitude spaced $300 \mathrm{~m}$ apart (Fig. 1-A). Samples of the rhizosphere soil, roots, rhizomes, and leaves were separately collected from each plant (Fig. 1-B). Rhizosphere soil was strictly defined as soil particles adhering to roots. To standardize and maximize the reproducibility of plant samples, $1 \mathrm{~g}$ of roots and $5 \mathrm{~g}$ of rhizomes were collected from each plant. For leaf samples, the same number of leaves and petioles were removed from each group of plants.

\section{Sample processing}

The samples were processed according to the method described by Guo et al. (Guo et al., 2000). Briefly, the excess soil was shaken from the roots, the soil that remained attached to the roots was defined as the rhizosphere soil. The root samples were separated from soil particles by shaking on a platform (10 $\mathrm{min}, 120 \mathrm{rpm} / \mathrm{min})$. The soil particles that directly dislodged from roots represented the rhizosphere soil. The rhizosphere soil was collected in an aseptic polyethylene (PE) tube and used for DNA extraction. The root, rhizome, and leaf samples were washed with flowing tap water to remove the excess soil; they were then rinsed with $75 \%$ alcohol for $3 \mathrm{~min}$ before being treated with 5\% hypochloric acid for $2 \mathrm{~min}$. Then, the plant samples were washed with sterile water three times to remove epiphytic microorganisms from the plant samples and obtain aseptic root, rhizome, and leaf samples. The rhizosphere soil, root rhizomes, and leaf samples were treated with liquid nitrogen ( $30 \mathrm{~s})$ before the total DNA was extracted with the corresponding DNA extraction kit. All sample materials were stored at $-80{ }^{\circ} \mathrm{C}$ before further treatment.

\section{DNA extraction}

DNA was extracted from all the rhizosphere soil, root, rhizome, and leaf samples. DNA extraction bias was minimized. Approximately $0.5 \mathrm{~g}$ of rhizosphere soil was used for each individual DNA 
114 extraction with the soil DNA extraction kit following the protocol provided by the manufacturer 115 (MoBio Power Soil DNA extraction kit 12888-50). Approximately $0.5 \mathrm{~g}$ of plant tissue samples 116 was used for each individual DNA extraction. The DNA of the plant tissue samples was extracted 117 using the magnetic bead method (genomic DNA extraction kit DP342) according to the 118 manufacturer's protocol.

\section{PCR amplification and sequencing}

The internal transcribed spacer (ITS) regions of the ribosomal RNA gene were amplified by PCR using the primers ITS1-1F-F CTTGGTCATTTAGAGGAAGTAA, ITS1-1F-R GCTGCGTTCTTCATCGATGC, ITS5-1737F GGAAGTAAAAGTCGTAACAAGG, and ITS22043R GCTGCGTTCATCGATGC. PCR was carried out of a $20 \mu \mathrm{L}$ mixture containing $4 \mu \mathrm{L}$ of $5 \times$ FastPfu buffer, $2 \mu \mathrm{L}$ of $2.5 \mathrm{mM}$ dNTPs, $0.8 \mu \mathrm{L}$ of primer $(5 \mu \mathrm{M}), 0.4 \mu \mathrm{L}$ of Fast Pfu polymerase and $10 \mathrm{ng}$ of template DNA. The amplification products were extracted from $2 \%$ agarose gel, and the AxyPrep DNA gel extraction kit (Axygen Bioscience, United City, CA, USA) was used. Purifications were carried out according to the manufacturer's instructions and quantified by QuantiFluor-St (Promega, USA).

The purified PCR products were measured by Qubit 3.0 (Life Invitrogen), and the average mixture of each of the 24 amplified fragments with different bar codes was obtained. The Illumina library was constructed by using polymerized DNA products according to the preparation process of the Illumina genomic DNA library. The amplified library was paired and sequenced on the Illumina MiSeq platform (Beijing Novosource Bioinformation Technology Co., Ltd) according to the standard protocol. The original data were stored in the National Center for Biotechnology Information (NCBI) sequence, and the archived (SRA: PRJNA504372) database is accessible via the link https://www.ncbi.nlm.nih.gov/sra/PRJNA504372.

137

138

139

140

141

142

143

144

145

146

147

148

149

150

151

152

153

\section{Sequence processing}

Using the analysis platform at our research centre, the raw sequence data were first screened by FastQC software to remove the low-quality sequences and then processed using USEARCH (version 11) (http://www.drive5.com/usearch/) for the subsequent bioinformatics analysis. The USEARCH process was carried out using the fastq_mergepairs method to merge the two terminal sequences. Primer excision was analysed using Cutadapt 1.18 (https://cutadapt.readthedocs.io/en/stable/). Usearchfastq_filter was used to control the sequence quality. The Fastx_uniques method was used to remove unnecessary and singleton sequences (the minimum parameter was 8). The unoise3 algorithm was used to cluster operational taxonomic units (OTUs) (sub-OTUs) without a reference, and the OTU table was generated by otutab. The clustered sequence was annotated by the Unite database, and the Syntax method had a cut-off value of 0.8. The OTU table was obtained after extraction using the least sequence value in the grouping.

\section{Statistical analysis}

Statistical analyses were performed using R 3.5.1 (The R Foundation for Statistical Computing, Vienna, Austria) (Edgar et al., 2010). Alpha and beta diversities were analysed using USEARCH. The ANOVA method and T-test method were used to study the $\alpha$-diversity through global diversity on the niches of the rhizosphere soil, root, rhizome and leaf samples. Beta diversity, through the 
154 Binary Jaccard distance method, was analysed by principal coordinate analysis (PCoA) and

155

156

157

158

159

160

161

162

163

164

165

166

167

168

169

170

171

172

173

174

175

176

177

178

179

180

181

182

183

184

185

186

187

188

189

190

191

hierarchical clustering analysis of UPGMA (Unweighted Pair-group Method with Arithmetic Mean), and the distances between different niches were measured. A Venn diagram was used to display the numbers of common and unique OTUs and the shared OTUs among different samples (Hanbo et al., 2011). The OTU sequence with the highest taxonomic abundance was selected as the representative sequence by Quantitative Insights Into Microbial Ecology (QIIME) software. Multiple sequence alignments were carried out, and the phylogenetic tree was constructed. The graph was prepared by using Python language.

\section{Results}

\section{Quality metrics of pyrosequencing analysis}

A total of 6159204 raw reads were obtained by sequencing. The raw data were submitted to NCBI under the accession number PRJNA504372. After quality trimming and assigning the raw reads to the corresponding samples, 4369448 clean reads remained in the dataset. There were 121373 clean reads per sample with an average length ( \pm standard deviation) of $222 \pm 50 \mathrm{bp}$ (Table 1-A). The readings that could not be clearly classified at the phylum level were also determined. These results showed that the number of OTUs that could not be classified was significantly higher in rhizosphere soil than in plants. The uncombined OTU number was lower in the plant compartments (roots, $16.68 \pm 9 \%$; rhizomes, $22.74 \pm 30 \%$; leaves, $28.23 \pm 12 \%$ ) than in the rhizosphere soils $(46.16 \pm 20 \%)$ (Table 1-B). All unclassified data were removed from the dataset before further analysis.

\section{Alpha rarefaction curves and alpha diversity}

Fig. 2 shows the constructed alpha rarefaction curves. The change in the rarefaction curve was lower for the endosphere samples than for the rhizosphere samples, and the endosphere fungal diversity was much lower than the rhizosphere diversity. Clustering was performed by unoise3, and the rarefaction curves for evaluating OTU richness were close to saturation. Most endosphere fungi tended to saturate at 210 330 OTUs in the rhizomes and 300 380 OTUs in the roots and leaves. Rhizosphere fungi in the rhizosphere soil were saturated at 750 1150 OTUs. Statistical differences in OTU richness were inferred from alpha diversity measures (Fig. 3). The rarefaction curve of rhizosphere soil began to flatten at $70 \%$, indicating that the depth of sequencing was sufficient to reliably describe the fungal communities associated with the plant compartments. The rhizosphere soil showed the highest fungal diversity, followed by the roots of Paris polyphylla Sm., and the rhizomes had the lowest relative abundance of fungi, possibly due to rhizome selectiveness for endophyte colonization. Additionally, the accumulation of plant secondary metabolites may have affected the abundance of endosphere fungi. Ding et al. and Wen et al. (Ding et al., 2018; Wen et al., 2015) studied secondary metabolites in different compartments of Paris polyphylla Sm. The types and contents of metabolites in different compartments of Paris polyphylla $\mathrm{Sm}$. are different.

\section{Alpha diversity}

Peer) reviewing PDF | (2019:07:39346:2:0:NEW 22 Dec 2019) 
192 The analysis of the OTU richness, Chao1 index, Shannon index, and Simpson diversity index

193

194

195

196

197

198

199

200

201

202

203

204

205

206

207

208

209

210

211

212

213

214

215

216

217

218

219

220

221

222

223

224

225

226

227

228

229

230

231

revealed the alpha diversity (microbial diversity) in each sample (Fig. 3-A, 3-B). The richness of rhizosphere soil was significantly higher than that of endosphere fungi (rhizosphere - root, $\mathrm{P}$ $=0.000007$; rhizosphere - rhizome, $\mathrm{P}=0.000001$; rhizosphere - leaf, $\mathrm{P}=0.000005)$. The richness of rhizosphere soil $(861 \pm 245)$, roots $(323 \pm 70)$ and rhizomes $(318 \pm 94)$ showed a decreasing trend among different samples. The OTU richness index was slightly higher in the leaves $(356 \pm 29)$ than in the rhizomes and roots. The Chao1 index also reflected a similar trend. According to the Shannon index and Simpson diversity index, the diversity of rhizosphere soil samples was higher than that of plant endosphere fungi, and the diversity of plant rhizomes was the lowest (Fig. 3-C, 3-D).

\section{Beta diversity}

Beta diversity was analysed at the OTU level, and the composition of fungal communities in different ecological niches were compared. The Binary Jaccard dissimilarity matrix was calculated using a PCoA to show the global similarity of fungal community structures in different samples (Fig. 4).

The PCoA showed that the rhizosphere soil communities were clustered at different phylogenetic levels, and no obvious clustering was found in the plant fungal communities. At the OTU level, principal component PC1 explained $27.74 \%$ of the total variation, and PC2 explained $15.99 \%$. Rhizosphere soil samples were well separated from the root, rhizome and leaf samples (Fig. 4-A). Hierarchical clustering of the samples was based on Binary Jaccard dissimilarity. Similarities based on Binary Jaccard (Fig. 4-B) were superimposed on the PCoA plot. For the plant samples, the microbes were not entirely clustered according to the intervals among different plant compartments (Fig. 4). These situations indicated that the microbial communities were similar among different plant compartments. To statistically support the visual clustering of the fungal communities in the above PCoA analyses, different plant compartments were examined using ANOSIM (an analogue of univariate ANOVA) with the Spearman rank correlation method (Table 2). All plant compartments exhibited fungal microbiota that were significantly dissimilar from each other (P values listed in Table 2) at the OTU level.

\section{Phylum and genus level differences in the fungal microbiome in different habitats}

We analysed the classification of fungi at the phylum and genus levels. A species analysis was also carried out and showed that there were 8 phyla in rhizosphere soil, 7 phyla in roots, 7 phyla in rhizomes, and 7 phyla in leaves. The fungal communities were successfully distinguished among the different soil/plant parts (rhizosphere soil, roots, rhizomes, and leaves) at the phylum level. The relative abundances of fungal taxa varied with plant compartment. The relative abundance of Basidiomycota was higher (26.19\%) in the rhizosphere soil than in plant parts (roots $16.61 \%$, rhizomes 10.16\%, and leaves 23.61\%). The proportion of Ascomycota was higher in plants $(\mathrm{P} \leq 0.01)$ than in rhizosphere soil $(15.35 \%)$. These results indicated that the plants had a selective enrichment preference for Ascomycota. The abundance of Ascomycota was also higher in rhizomes $(85.89 \%)$ than in other plant parts, possibly because rhizomes are the primary part of the plant that produces medicinal components. The role of Ascomycota in the synthesis of active

Peer) reviewing PDF | (2019:07:39346:2:0:NEW 22 Dec 2019) 
232 components needs further study. Glomeromycota also occupied a certain proportion in the parts of 233 the plants (roots $12.14 \%$, rhizomes $2.82 \%$, and leaves $3.50 \%$ ), while its enrichment in soil was

234

235

236

237

238

239

240

241

242

243

244

245

246

247

248

249

250

251

252

253

254

255

256

257

258

259

260

261

262

263

264

265

266

267

268

269

270

271 only $0.01 \%$. This result indicated that the plant parts had a preference for Glomeromycota.

At the genus level, 72 genera were annotated in rhizosphere soils, 75 in roots, 80 in rhizomes, and 78 in leaves. The distribution of the top 10 genera was also different among the rhizosphere soil, roots, rhizomes, and leaves. In the rhizosphere soil, except in the unassigned soil, Clavulina was found to be the most abundant genus (11.29\%). The enrichment of Clavulina was less than $1 \%$ in both plant parts (roots $0.07 \%$, rhizomes $0.002 \%$, and leaves $0.03 \%$ ) and rhizosphere soil. The enrichment of Cadophora was less than $1 \%$ in rhizosphere soil but much higher in plant parts (roots, $6.06 \%$; rhizomes, $24.84 \%$; and leaves, $2.53 \%$ ). The relative abundance of Cadophora was in the order of rhizome $>$ root $>$ leaf in the plant compartments, which was consistent with the results at the phylogenetic level; Cadophora belongs to Ascomycota. Ascomycota is considered the most likely endophytic taxon to colonize plants (Guo et al., 2016). Ascomycota is widely found in soils and in plants such as forage grass, flowers, and crops (Hacquard et al., 2015, Edwards et al., 2015).

In addition, the distribution of genera and phyla of the different fungal communities (rhizosphere soil, roots, rhizomes and leaves) among different altitudes was analysed. As shown in Fig. 5-B, at the phylum level, the fungal phyla at different altitudes showed different trends. In the rhizosphere soil, the number of unassigned OTUs increased gradually with increasing altitude, which is consistent with previously reported results (Guo et al., 2000). In addition, the microbiome also varied among different plant compartments. High enrichment of Ascomycota was found in the plants at the low-altitude areas, especially in the roots $(91.42 \%)$ and leaves $(82.54 \%)$. However, in the rhizomes of plants, the relative abundance of Ascomycota was higher (95.09\%) in the middlealtitude area than in the other areas. Basidiomycota, another important phylum in plants, also varied with the altitude gradient. In the roots and leaves, Basidiomycota showed an increasing trend with increasing altitude, but such a trend was not found in the rhizomes of plants. The fungi also showed different trends at the genus level (Fig. 5-D). In rhizosphere soil, the annotated OTUs showed a downward trend with increasing elevation to $1800 \mathrm{~m}$ (only 14.95\%). The dominant mycobiota, such as Tetracladium and Clavulina, also showed decreasing trends with increasing elevation. The number of annotated OTUs in each plant was higher in the middle-altitude area than in the low and high-altitude areas (roots 70.07\%, rhizomes 67.63\%, and leaves $75.85 \%$ ). Tetracladium also decreased in abundance with increasing altitude. Cadophora, with a high enrichment rate in plants, showed different trends in different flora. The enrichment of Cadophora in rhizomes was greatest at the middle altitude (46.17\%). To obtain a complete overview of OTU distribution in different plant compartments, we calculated the OTUs in each particular plant compartment as well as the OTUs shared among the different plant compartments (Fig. 6). R software was used to draw a Venn diagram (Caporaso et al., 2010). The proportion of exclusive OTUs in rhizosphere soil was $22.89 \%$, and the proportion of OTUs in plants was $34.15 \%(0.18 \%$ in the roots, $0.35 \%$ in the rhizomes, and $0.18 \%$ in the leaves). The overlap rate of OTUs between rhizosphere soil and the root and rhizome samples was $38.73 \%$ and $39.79 \%$, respectively. The 
272 overlap rate between rhizosphere soil and leaf samples was $35.56 \%$. The proportion of OTUs 273 simultaneously detected in rhizosphere soil and plants was $42.96 \%$.

274 The annotated microbial communities in the rhizosphere soil were mainly composed of 275 Clavulina, Tetracladium, Ceratobasidium and Aureobasidium. These genera and Rhizophagus 276 constituted the dominant microorganism community in plants, with extremely varied ecological 277 niches. The dominant genera in the root samples were Tetracladium, Aureobasidium, and 278 Cadophora, but the dominant genera in the rhizome samples were Cadophora, Tetracladium and 279 Ceratobasidium. The leaf samples mainly contained Aureobasidium, Alternaria, Kluyveromyces, 280 Cladosporium and Tetracladium. These microbes have been isolated from a large variety of plants 281 and have considerable promoting effects on plant health and growth (Raven et al., 1970; Gottel et 282 al., 2011). Cadophora and Tetracladium were found to effectively colonize and enrich Paris 283 polyphylla $\mathrm{Sm}$., with significant differences in relative abundance in roots $(6.06 \%$ and $20.82 \%$, 284 respectively), rhizomes $(24.84 \%$ and $8.46 \%$, respectively) and leaves $(2.53 \%$ and $4.39 \%$, 285 respectively).

286 Cluster analysis of microbiome abundance in rhizome

287 As the rhizome is the medicinal part of the plant, we analysed the species abundance of microbes 288 in the rhizomes. The medicinal parts of wild rhizomes were analysed at different altitudes. Cluster 289 heat maps of species (Fig. 7-A) were developed on the basis of species composition and the relative 290 abundance of each sample. All data of the samples at the same altitude were combined and mapped 291 using the R tool. The core mycobiota at the genus level were also analysed (Fig. 7-B). Cadophora 292 was the core fungal microbiome in the rhizome.

293 After analysis with QIIME software, the sequence of OTUs for the most abundant genus in 294 the rhizome compartment was selected as the reference sequence to perform multiple sequence 295 alignments and construct the phylogenetic tree (Fig. 8). We defined five phyla with high species 296 abundance in the rhizomes. Ascomycota formed a large independent branch, and its species 297 abundance presented clear advantages over other phyla. This result further confirms the floristic 298 selectivity of the host.

\section{Discussion}

300 Quality of the pyrosequencing analysis

301 We used ITS1-1F and ITS1-5F primer mixtures to maximize the phylogenetic coverage of fungi.

302 The high abundance of chloroplast genes can lead to unexpected co-amplification of non-target 303 sequences (Kabir et al., 2016; Roesch et al., 2007). In this study, the amplified plant chloroplast 304 genes were culled. Remarkably, the singletons in rhizosphere soil and in different plant 305 compartments had been removed before annotation. Due to the structural differences between soil 306 and plants, we were unable to extract high-quality and high quantity DNA from all plant samples 307 using the same DNA extraction kit. To ensure high-quality and high quantity DNA from all studied 308 samples, we chose different pre-processing methods, and extraction kits were used for DNA 309 extraction from rhizosphere soil and plant samples. As a result, the high discrepancy in the number 310 of singletons in the plant parts could be attributable to genuinely rare (singleton) OTUs in the 
311 rhizosphere soil (Table 1). In fact, microbiomes in the rhizosphere soil are generally considered 312 the most diverse regions (Lugtenberg et al., 2009; Coleman et al., 2016). For further analysis, we 313 chose to remove all singletons from the data sets. However, the involvement role of singletons 314 ecological roles functions is largely unknown, this requires need the further study.

315 Endophytic fungal communities in different niches

316 We estimated the richness, evenness and diversity of alpha diversity based on OTUs. We found 317 that the abundances of OTUs in rhizosphere soil and plant compartments (roots, rhizomes and 318 leaves) were clearly different. The results are consistent with the general view of endosphere 319 colonization. The diversity and evenness varied greatly from rhizosphere soil to endosphere. Because of chemotaxis and colonization of the rhizosphere microbiome, rhizosphere microbial communities are rich and diverse (Huang et al., 2018; Bulgarelli et al., 2012; Hardoim et al., 2008). The rhizosphere soil-root interface acts as a selective barrier for endosphere fungal colonization. High variability of endophytic OTU richness, as depicted by the box plot, could possibly be caused by sporadic and nonuniform colonization of the roots, rhizomes and leaves of Paris (Gottel et al., 2011; Beckers et al., 2017). Therefore, our data suggest considerable variation in endophytic colonization as a major reason for the high variability in the box plot. At the genus level, Cadophora and Tetracladium were the dominant microorganisms among the plant mycobiota.

328

329

330

331

332

333

334

335

336

337

338

339

340

341

342

343

344

345

346

347

348

349

350 Although their OTU abundance was abundances were different, both Cadophora and Tetracladium belong to Ascomycota, which is considered the most likely endophytic taxon to colonize plants (Guo et al., 2016). Ascomycota is widespread in soil and plants, such as forage, flowers and crops (Kottke et al., 2008; Edwards et al., 2015; Hacquard et al., 2015). Enrichment and depletion of specific microbiomes within the plant-associated microbiome are initiative processes that depend on active selection of microbial consortia by the plant host and opportunistic colonization of the available ecological niches (Bulgarelli et al., 2013; Mehta et al., 2001; Wen et al., 2007). Therefore, the colonized fungi are limited to specific fungal species. Our results illustrated that the diversity and evenness decreased from the rhizosphere to the endosphere. Only a limited number of microbes could adapt to the way of life in the plant. As a result, the plant niches consist of specific endosphere communities. Previous studies of other plants have also reported niche differences in the distribution of endogenous microbial communities, such as poplar (Beckers et al.,2017, Cregger et al., 2018), which may have an effect on plant metabolism (Chen et al., 2018). Different niches of the plant may be associated with differences in plant metabolism. Studies of the differences in the endogenous microbial community in different niches could provide a basis for future studies of differences in metabolism in different parts of medicinal plants.

To compare the structure of the endosphere fungal community in different plant compartments, we clustered all samples utilizing PCoA and hierarchical clustering (BinaryJaccard) (Fig. 4). At the OTU level, the rhizosphere soil gathered together. However, there was no obvious relationship between the fungal microorganism clusters in different plant tissues. This result was further confirmed by UPGMA hierarchical cluster analysis. Paris polyphylla $\mathrm{Sm}$. is a perennial herb. In each growth cycle of Paris polyphylla Sm., only the aboveground part of the plant dies, while the rhizome remains alive. This growth pattern may be one reason for the lack of 
351

352

353

354

355

356

357

358

359

360

361

362

363

364

365

366

367

368

369

370

371

372

373

374

375

376

377

378

379

380

381

382

383

384

385

386

387

388

389

390

obvious typical mycobiota in different plant compartments. In addition, for microorganisms, the endophytic environment of plants is complex, In contrast to rhizosphere colonization, intricate interplay between endophytic microbe and the host plants innate immune system, it of the host plant is completely different from the soil, which is also an important reason for the difference between the soil and plant microbial communities.

In this study, different rarefaction curves (Fig. 2) were obtained from the rhizosphere soil and endosphere samples. Compared with plants, the rhizosphere soil displayed much greater microbial diversity, possibly because the plant provides a relatively stable interior environment, which leads to lower variability in the plant's internal fungal community. In addition, we found that the OTU numbers in soil were almost three times those in plants. This result is consistent with the widely accepted view that soil contains many microbes. The structure of the endosphere fungal communities varied more markedly than the structure of the rhizosphere community (Bulgarelli et al., 2012; Nallanchakravarthula et al., 2014). Soil microbe communities form one of the most abundant microbial ecosystems on Earth (Coleman et al., 2016; Wu et al., 2005). In addition, root exudates and nutrients from mucilage sources also attract countless organisms to gather in the rhizosphere. The microbes in plants need to be strongly competitive to successfully colonize roots (Hackstein et al., 2010); competitive ability might manifest as the ability to break through plant cells (producing enzymes that degrade cell walls) and the ability to adapt to the innate immunity of plants (Turner et al., 2013; Compant et al., 2010; Nie et al., 2005).

\section{Differences in the microbiome of plants among different altitudes}

The content of secondary metabolites from the same medicinal plant species can vary depending on the location of cultivation, which could in part be related to differences in the composition of the associated microbial communities at different sites. According to Köberl et al. (2013), many of the variations in the quality of traditional herbal medicine may be attributable to changes in the microbial community either in the rhizosphere or in the endophytic compartment of the medicinal plant (Huang et al., 2018). The functional characteristics of endophytic communities residing inside the roots of rice have revealed that endophytes may be involved in the metabolic processes of rice (Vain et al., 2014). Salvia miltiorrhiza harbours a distinctive microbiome that is enriched in functions related to secondary metabolism and thus may contribute additional metabolic capabilities beyond those encoded in the genome of the host plant (Huang et al., 2018, Chen et al., 2018). These observations suggest that the soil and climate at different locales can influence the metabolite content of the medicinal plant. Altitude is one of the most important factors affecting climate, therefore we analysed the variation in the endophytic fungal communities among different altitudes. We divided the plant distribution into three elevation zones: low, middle and high altitude (Fig. 5). The results showed downward trends of the richness and diversity of soil microorganisms in the rhizosphere with increasing altitude. Previous studies have shown that fungal community composition is influenced by both ecological factors and evolutionary factors. Spatial scale is considered a major factor contributing to differences in fungal diversity (Kabir et al., 2016). However, the richness and diversity of endophytic microorganisms do not follow the distribution law of soil microorganisms. In the endophytic environment, the richness and diversity

Peer) reviewing PDF | (2019:07:39346:2:0:NEW 22 Dec 2019) 
391

392

393

394

395

396

397

398

399

400

401

402

403

404

405

406

407

408

409

410

411

412

413

414

\section{5}

416

417

418

419

420

421

422

423

424

425

426

427

of endophytic microorganisms are the most abundant in the plant endophytic environments, which is similar to the distribution law of plants, rather than following the law of altitude. This phenomenon has rarely been reported in previous studies. Through systematic study of the associated microbiome in medicinal plants, we should be able to clarify the distribute situation of various microbes in plants at different elevations. This information could guide better selection of growing environments for the cultivation of medicinal plants, which in turn may improve the medicinal quality and evaluation standards of medicines that will facilitate their passing more rigorous scientific and commercial evaluations.

\section{Conclusions}

This study revealed the structural variability and niche differentiation in the rhizosphere and endosphere fungal microbiomes of wild Paris plants at different altitudes. The results show that the structural variability in microbiome communities in the rhizosphere soil is lower in wild Paris polyphylla $\mathrm{Sm}$. than in endosphere fungal communities. The formation of rhizosphere fungal communities is a stable process, and endophytic colonization is variable. In addition, our data confirm reports of niche differentiation in rhizosphere soil-root microbiome communities. Furthermore, our study not only reveals the relationships and differences in endosphere fungal communities in various plant tissues but also clearly shows relationships between altitude and the endosphere microbiome in plants. With increasing altitude, the diversity of the plant endosphere microbiome first increased and then decreased; this pattern is inconsistent with the relationship generally observed between soil microorganisms and altitude. In addition, the core members of the endophytic microbial communities in the rhizome of Paris polyphylla Sm. were successfully identified. The present findings provide a basis for further studies on the interactions between the endosphere microbiome and hosts and can inform efforts involving the artificial planting of this plant.

\section{Acknowledgements}

The authors would like to thank ShiQiang Cheng, MingZhong Hao, QiLing Sun, and DanNi Zhu for their assistance with the field work.

\section{References}

Bao, Y.R., Liu, Y., Xie, S.X., Xu, Y.J., 2017. Study on the effect of endophytic fungi on active constituents of medicinal plants. Ginseng Research 5, 40-46.

Beckers, B., Beeck, M., Weyens, N., Boerjan, W., Vangronsveld, J., 2017. Structural variability and niche differentiation in the rhizosphere and endosphere bacterial microbiome of fieldgrown poplar trees. Microbiome 5, 25-42.

Bodenhausen, N., Horton, M.W., Bergelson, J., 2013. Bacterial communities associated with the leaves and the roots of Arabidopsis thaliana. PLoS One 8, 5632-5639.

Bulgarelli, D., Rott, M., Schlaeppi, K., Ver Loren van Themaat, E., Ahmadinejad, N., Assenza, F., Rauf, P., Huettel, B., Reinhardt, R., Schmelzer, E., Peplies, J., Gloeckner, F.O., Amann, R., 
428

429

430

431

432

433

434

435

436

437

438

439

440

441

442

443

444

445

446

447

448

449

450

451

452

453

454

455

456

457

458

459

460

461

462

463

464

465

466

467

Eickhorst, T., Lefert, P.S.,2012. Revealing structure and assembly cues for Arabidopsis rootinhabiting bacterial microbiota. Nature 488, 91-95.

Bulgarelli,D., Schlaeppi, K., Spaepen, S., Ver Loren van Themaat, E., Schulze, L. P., 2013. Structure and functions of the bacterial microbiota of plants. Annu Rev Plant Biol. 64, 807838.

Caporaso, J.G., Kuczynski, J., Stombaugh, J., Bittinger, K., Bushman, F.D., Costello, E.K., Fierer, N., Peña, A.G., Goodrich, J.D., Gordon, J.I., Huttley, G.A., Kelley, S.T., Knights, D., Koenig, J.E., Ley, R.E., Lozupone, C.E., Mcdonald, D., Muegge, B.D., Pirrung, M., Reeder, J., Sevinsky, J.R., Turnbaugh, P.J., Walters, W.A., Widmann, J., Yatsunenko, T., Zaneveld,J., Knight, R., 2010. QIIME allows analysis of high throughput community sequencing data. Nature methods. 7, 335-336.

Chen, H.M., Wu, H., Yan, B., Zhao, H., Liu, F., Zhang, H., Sheng, Q., Miao, F., Liang, Z., 2018. Coremicrobiome of medicinal plant Salvia miltiorrhiza seed: a rich reservoir of beneficial microbes for secondary metabolism? International journal of molecular sciences 19, 672-686.

Coleman, D.D., Desgarennes, D., Fonseca, G.C., Gross, S., Clingenpeel, S., Woyke, T., North, G., Axel, V.A., Partida, M.L.P., Tringe, S.G., 2016. Plant compartment and biogeography affect microbiome composition in cultivated and native Agave species. New Phytol. 209, 798-811.

Compant, S., Clément, C., Sessitsch, A., 2010. Plant growth-promoting bacteria in the rhizosphere and endosphere of plants: their role, colonization, mechanisms involved and prospects for utilization. Soil Biol Biochem. 42, 669-678.

Cregger, M.A., Veach, A.M., Yang, Z.K., Crouch, M.J., Vilgalys, R., Tuskan, G.A., Schadt, C.W., 2018. The Populus holobiont dissecting the effects of plant niches and genotype on the microbiome. Microbiome. 6, 31-45.

Ding, L.S., Zhao, M., Li, Y.M., Chen, L.M., Wang, Z.M., Wang, Z.J., Gao, H.M., Li, S.Q., 2018. Study on the Anti-nociceptive and Anti-inflammatory Effects of The Extract of Aerial Part and Rhizome of Paris polyphylla var. Chinensis Nat Prod Res Dev. 30, 832-839.

Edgar, R.C., 2010. Search and clustering orders of magnitude faster than BLAST. Bioinformatics. 26, 2460-2461.

Edwards, J., Johnson, C., Santos-Medellín, C., Lurie, E., Podishetty, N.K., Bhatnagar, S., Eisen, J.A., Sundaresan, V., 2015. Structure variation and assembly of the root-associated microbiomes of rice. Proc Natl Acad Sci. 112, 911-920.

Gottel, N.R., Castro, H.F., Kerley, M., Yang, Z., Pelletier, D.A., Podar, M., Karpinets, T., Uberbacher, E., Tuskan, G.A., Vilgalys, R., Doktycz, M.J., Schadt C.W., 2011. Distinct microbial communities within the endosphere and rhizosphere of Populus deltoides roots across contrasting soil types distinct. Appl Environ Microbiol. 77, 5934-5944.

Guo, L.D., Hyde, K.D., Liew, E.C.Y., 2000. Identification of endophytic fungi from Livistona chinensis based on morphology and rDNA sequences. New Phytologist. 147, 617-630.

Guo, S.X., Endophytic mycology of medicinal plants [M]. 2016. ISBN: 9787030476852.

Hackstein, J.H.P., 2010. Evolution of prokaryote animal symbiosis from a genomics perspective. microbiology monographs, Springer. 207-233.

Peer) reviewing PDF | (2019:07:39346:2:0:NEW 22 Dec 2019) 
468

469

470

471

472

473

474

475

476

477

478

479

480

481

482

483

484

485

486

487

488

489

490

491

492

493

494

495

496

497

498

499

500

501

502

503

504

505

506

Hacquard, S., Garrido-Oter, R., González, A., Spaepen, S., Ackermann, G., Lebeis, S., McHardy, A.C., Dangl, J.L., Knight, R., Ley, R., Lefert, P.S., 2015. Microbiota and host nutrition across plant and animal kingdoms. Cell Host Microbe. 17, 603-616.

Hanbo, C., Paul, C.B., 2011. Venn Diagram, a package for the generation of highly customizable Venn and Euler diagrams in R. BMC Bioinformatics. 12, 35-45.

Hardoim, P.R., Van, O.L.S., Van, J.D., 2008. Properties of bacterial endophytes and their proposed role in plant growth. Trends Microbiol. 16, 463-471.

Huang, W.J., Long, C.L., Lam, E., 2018. Roles of Plant Associated Microbiota in Traditional Herbal Medicine. Trends in Plant Science. 23, 559-62.

Huang, Z.J., Guo, Z.Y., Yang, R.Y., She, Z.G., Lin, Y.C., 2007. A lkaloid metabolites of mangrove endophytic fungus ZZF42 from the South China Sea. Journal of Chinese Medicinal Materials. 08, 939-41.

Jones, J.D.G., Dangl, J.L., 2006. The plant immune system. Nature. 444, 323-329.

Kabir, G.P., Peter, G.K., Jennifer, M.T., 2016. Dimensions of biodiversity in the Earth Mycobiome. Nature Reviews Microbiology. 6, 59-68.

Kau, A.L., Ahern, P.P., Griffin, N.W., Goodman, A.L., Gordon, J.I., 2011. Human nutrition, the gut microbiome and the immune system. Nature. 474, 327-36.

Khan, A.L., Hanmayun, M., Kim, Y.H., Kang, S.M., Lee, J. H., Lee, I.J., 2011. Gibberellins producing endophytic Aspergillus fumigates sp. LH02 influenced endogenous phytohormonal levels, isoflavonoids production and plant growth in salinity stress. Procss Biochemistry. 46, 440-447.

Khan, A.L., Hanmayun, M., Kim, Y.H., Kang, S.M., Lee, I.J., 2011. Ameliorative symbiosis of endophyte (Penicillium funiculosum LHL06) under salt stress elevated plant growth of Glycine max L. Plant physiology and Biochemistry. 9, 852-861.

Khau, A.L., Ahern, P.P., Griffin, N.W., Goodman, A.L., Gordon, J.I., 2011. Human nutrition the gut microbiome and the immune system. Nature. 474, 327-336.

Kottke, I., Haug, I., Setaro, S., Suárez, J.P., Michael, W., Markus, P., Nebel, M., Oberwinkler, F., 2008. Guilds of mycorrhizal fungi and their relation to trees ericads orchids and liverworts in a neotropical mountain rain forest. Basic and applied ecology. 9, 13-23.

Köberl, M., Schmidt, R., Ramadan, E.M., Bauer, R., Berg. G., 2013. The microbiome of medicinal plants: diversity and importance for plant growth, quality, and health. Front Microbiol, 4, 400.

Lebeis, S.L., Paredes, S.H., Lundberg, D.S., Breakfield, N., Gehring, J., McDonald, M., 2015. Salicylic acid modulates colonization of the root microbiome by specific bacterial taxa. Science. 349, 860-864.

Lee, Y.K., Mazmanian, S.K., 2010. Has the microbiota played a critical role in the evolution of the adaptive immune system. Science. 330, 1768-1773.

Lu, L.W., Bao, S.S., Tan, M.H., Li, X.S., Lv, M.M., Liu, C.X., Deng, Z.S., Guo, Z.Y., Zou, K., 2018. Research on alkaloid metabolites from endophytic fungus penicillium sp. J of China Three Gorges Univ.(Natural Sciences). 40, 97-99. 
507 Lugtenberg, B., Kamilova, F., 2009. Plant growth promoting rhizobacteria. Annu Rev Microbiol. $508 \quad 63,541-556$.

509 Lundberg, D.S., Yourstone, S., Mieczkowski, P., Jones, C.D., Dangl, J.L., 2013. Practical 510 innovations for high-throughput amplicon sequencing. Nature Methods. 10, 999-1002.

511 Mehta, A., Rosato, Y.B., 2001. Differentially expressed proteins in the interaction of Xanthomonas 512 axonopodis pv. Citri with leaf extract of the host plant. Proteomics. 1, 1111-1118.

513 Nie, L.Y., Chen, L., Ren, A.Z., Gao, Y.B., 2005. Isolation and identification of Endophytes in 514 Lolium perenne L. Microbiology China. 32, 10-15.

515 Raven, P.H., 1970. A multiple origin for plastids and mitochondria, many independent symbiotic 516 events may have been involved in the origin of these cellular organelles. Science. 169, 641517646.

518 Roesch, L.F.W., Fulthorpe, R.R., Riva, A., Casella, G., Hadwin, A.K.M., Kent, A.D., Daroub, 519 S.H., Camargo, F.A., Farmerie, W.G., Triplett, E.W., 2007. Pyrosequencing enumerates and 520

521

522

523

524

525

526

527

528

529

530

531

532

533

534

535

536

537

538 contrasts soil microbial diversity. ISME J. 1, 283-290.

Srivathsa, N., Shahid, M., Sadhna, A., Roger D.F., 2014. Influence of soil type, cultivar and Verticillium dahliae on the structure of the root and rhizosphere soil fungal microbiome of strawberry. PloS one. 9, 10.

Turner, T.R., James, E.K., Poole, P.S., 2013. The plantmicrobiome. Genom Biol. 14, 1-10.

Vain, T., Crowell, E. F., Timpano, H., Biot, E., Desprez, T., Mansoori, N., 2014. The cellulase KORRIGAN is part of the cellulose synthase complex. Plant Physiol. 165, 1521-1532.

Wen, F.S., Van E.H.D., Tsaprailis, G., Hawes, M.C., 2007. Extracellular proteins in pea root tip and border cell exudates. Plant physiol. 143, 773-783.

Wen, Y.S., Geng, Y.Y., Wang, J.M., Hua, Y., 2015. Chemical Constituents of the Fibrous Roots of Paris polyphylla var. yunnanensis. Journal of West China Forestry Science. 6, 51-54.

Wu, J.R., Han, S.F., Zhu, Y.Y., Lv, M, Wang, G.P., Guo, W.L., 2005. Study on Taxonomy of Endophytic Fungi isolated from orchid mycorrhizae in Yunnan Province. Journal of southwest forestry college. 26, 5-10.

Xue, P.H., 2013. The study on the Biological activity of secondary metabolites from three medicinal plant endophytic fungi and one Tibetan medicinal plant. Lanzhou university of technology.

Yadav, K., Aggar, W.A., Singh, N., 2013. Arbuscular mycorrhizal fungi (AMF) induced

539 acclimatization, growth enhancement and colchicine content of micropropagated Gloriosa superba L. Plantlets. Industrial Crops and Products. 45, 88-93. 
Figure 1

Diagram of the sampling operation.

A: Sampling locations. Sampling was carried out at three different heights, $1200 \pm 50 \mathrm{~m}$, $1500 \pm 50 \mathrm{~m}$, and $1800 \pm 50 \mathrm{~m}$, with 3 plants at each altitude at an interval of $300 \mathrm{~m}$. B: Sampled parts of the plant. For each plant, samples were separately collected in the rhizosphere soil, roots, rhizomes, and leaves.
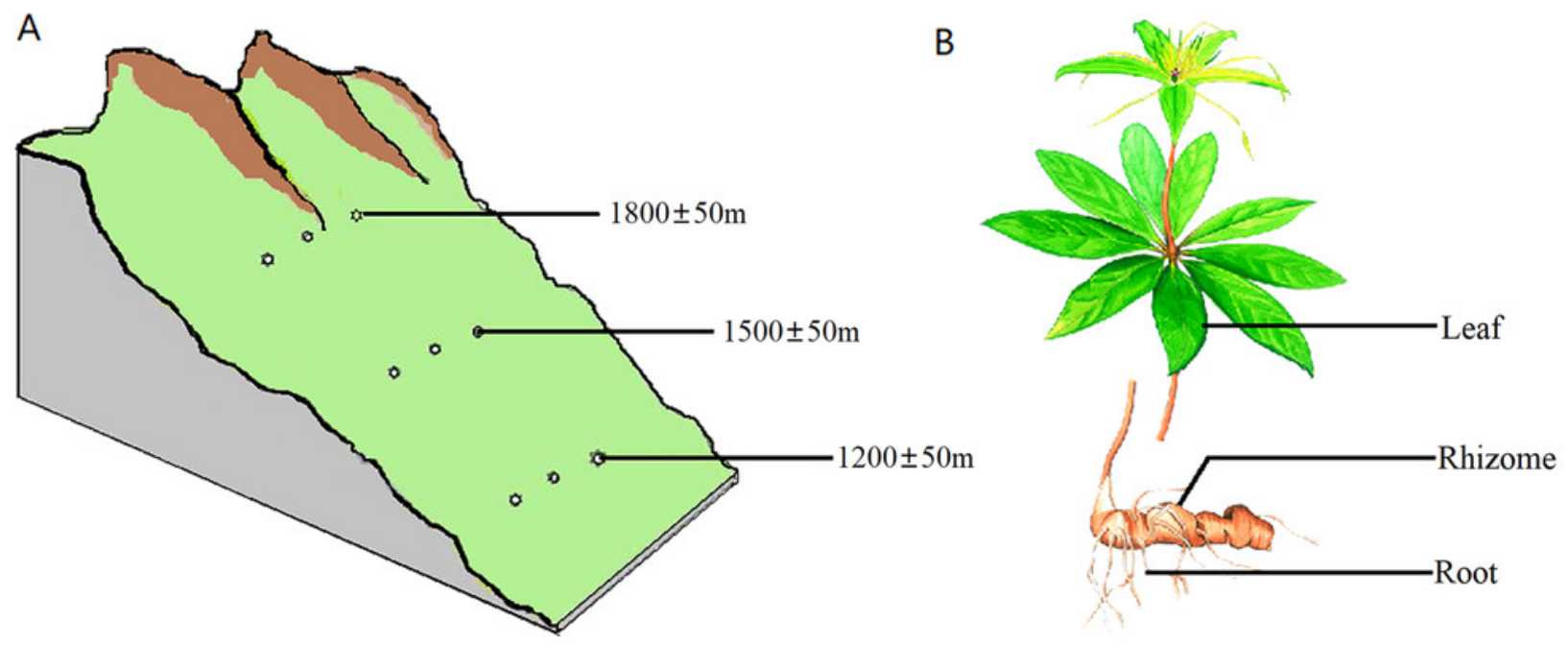


\section{Figure 2}

Rarefaction curves of all samples for each group.

Rarefaction curves of all samples for each group (rhizosphere soil, roots, rhizomes, and leaves). Clustering was performed by unoise 3. Each curve represents the average of all repeats ( \pm standard deviation) for each group of samples (rhizosphere soil, root, rhizome, and leaf). The rarefaction curve of rhizosphere soil began to flatten at $70 \%$, indicating that the depth of sequencing was sufficient to reliably describe the fungal communities associated with the plant departments.

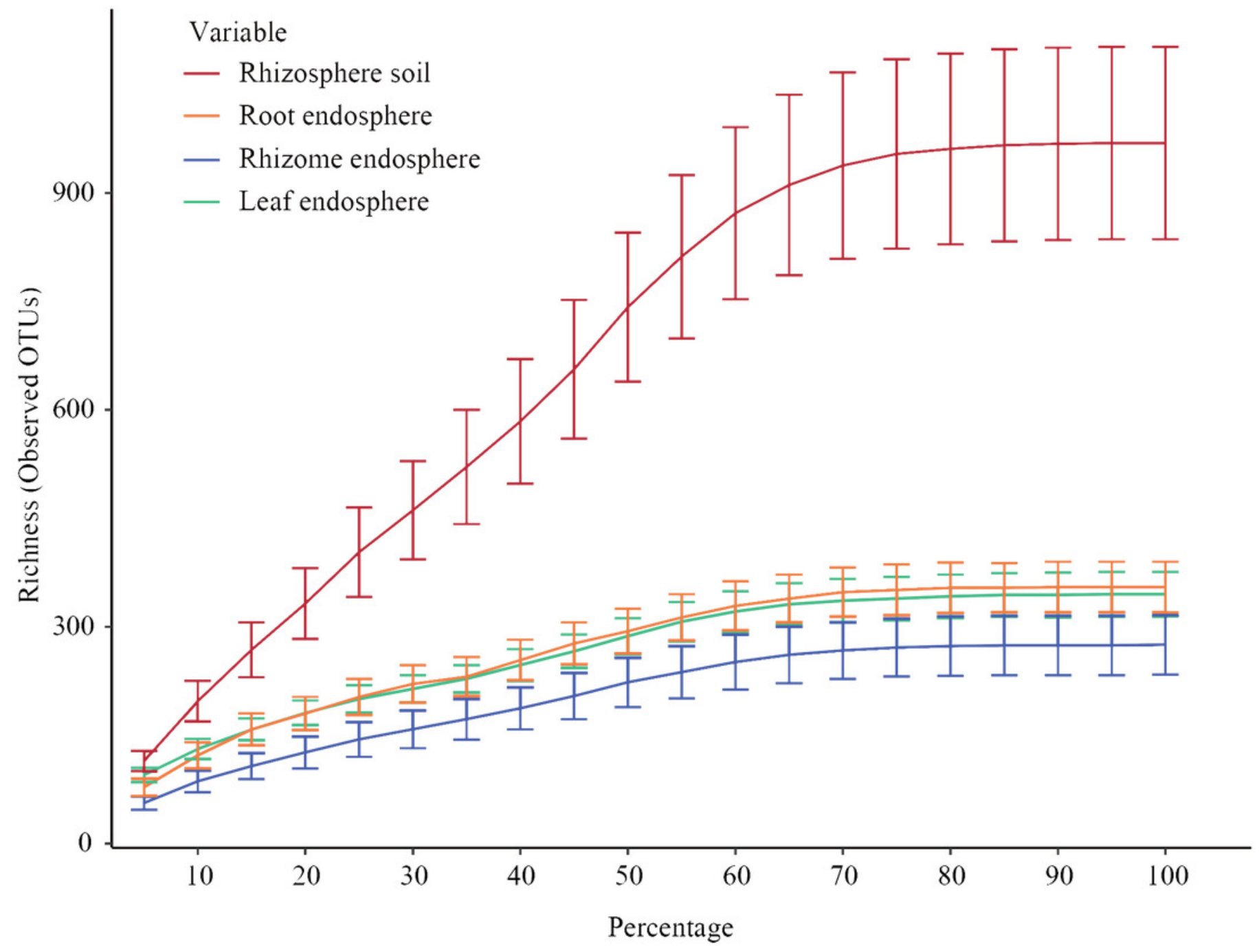




\section{Figure 3}

Alpha diversity of the fungal communities in different samples.

A: OTU richness estimates (number of observed OTUs). B: Chaol index. C: Shannon index. D: Simpson diversity index. The box diagram shows the first (25\%) and third (75\%) quartiles, median values and maximum and minimum observational values in each data set. The alpha diversity estimation is presented for the samples of rhizosphere soil, roots, rhizomes and leaves. The overall plant compartment $\mathrm{P}$ value is displayed at the top of each graph.

Significant differences $(P<0.05)$ across plant compartments are indicated with lowercase letters. 

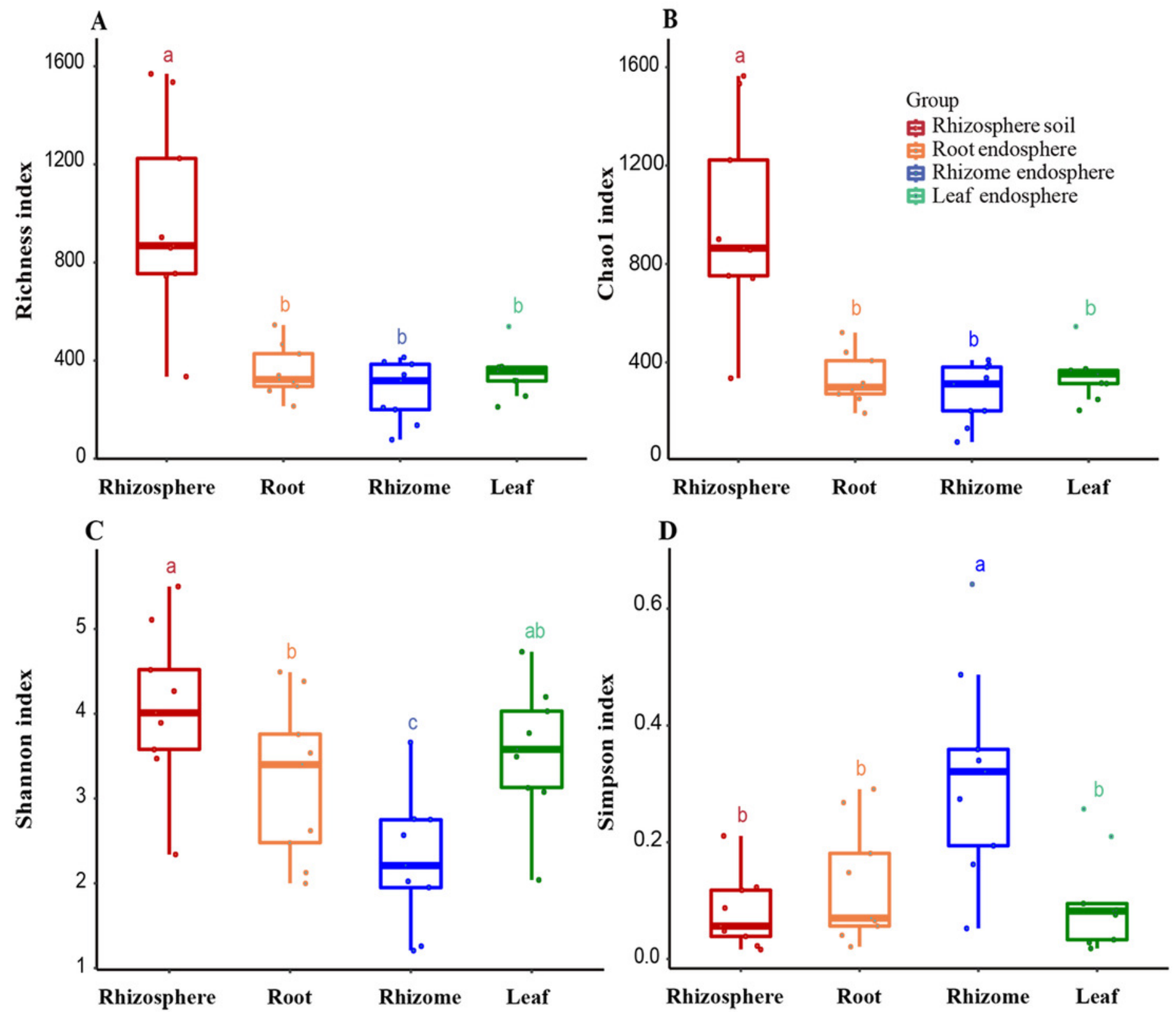


\section{Figure 4}

Plant compartment drives the composition fungal communities at the OTU level.

A: The horizontal and vertical coordinates are the two eigenvalues that lead to the greatest difference between samples, and the main influence degree is reflected as a percentage. PCOA to show the global similarity of fungal community structures in different samples OTUs differentiating the plant compartents are displayed as vectors on the PCoA plots. Rhizosphere soil samples were well separated from the plant comparts samples. B: Based on the PCoA of the binary-jaccard algorithm and hierarchical clustering analysis of UPGMA ( Unweighted Pairgroup Method with Arithmetic Mean ), t he similarity of species composition between samples, hierarchical clustering of the different samples based on binary-jaccard is determined. The closer the sample is, the shorter the branch length is, indicating that the species composition of the two samples is more similar. Similarities based on binary-jaccard were superimposed on the PCoA plot. 
A

PCoA - PC1 vs PC2

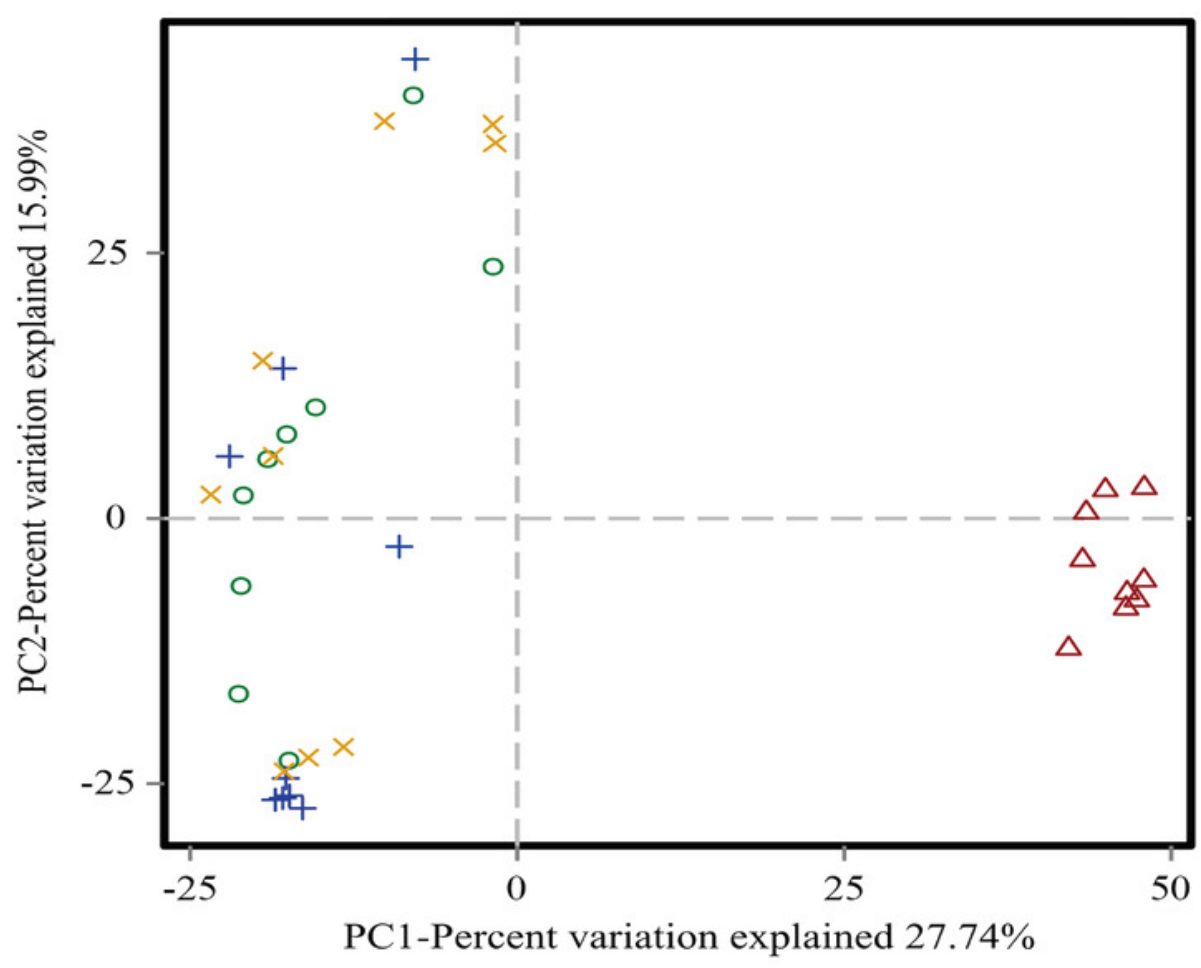

$\triangle$ Rhizosphere soil

$\times$ Root endosphere

+ Rhizome endosphere

- Leaf endosphere

B

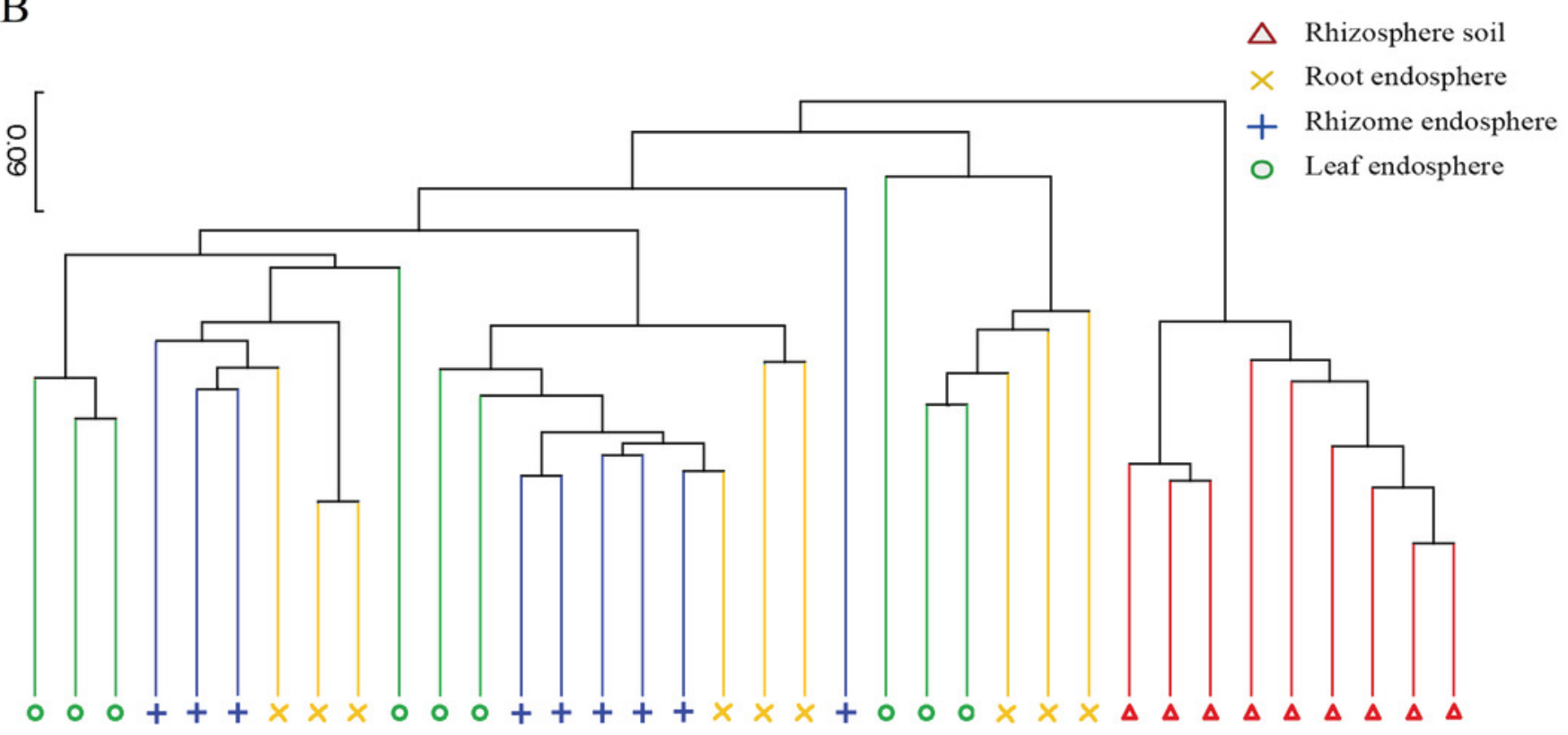




\section{Figure 5}

Distribution of OTUs at the phylum and genus levels.

A: The fungi phyla at different niches. B: The fungi phyla at different altitudes show different trends at the phylum level. C: The fungal genus at different niches. D: The fungal genus at different altitudes showed different trends at the genus level. For the best display, only the top 10 species of abundance are displayed, and the other species are merged into "Others". Unclassified represents the species that have not been annotated by taxonomy. Major contributing phyla are displayed in different colours, and the unannotated are grouped and displayed in greyish blue. L: low altitude $1200 \mathrm{~m}$. M: middle altitude $1500 \mathrm{~m}$. H: high altitude $1800 \mathrm{~m}$. Coverage at the phylum levels: The percentages of the total community covered by the core OTUs were $46.16 \%$ (rhizosphere soil), $96.76 \%$ (roots), 99.19\% (rhizomes) and $90.86 \%$ (leaves). Coverage at the genus level: The percentages of the total community covered by the core OTUs were $40.34 \%$ (rhizosphere soil), $52.20 \%$ (roots), $47.17 \%$ (rhizomes) and $56.66 \%$ (leaves). 
A

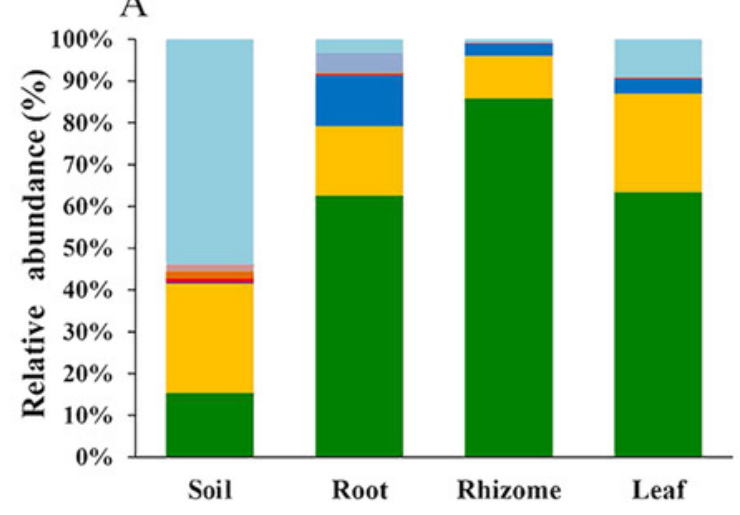

$\mathrm{C}$

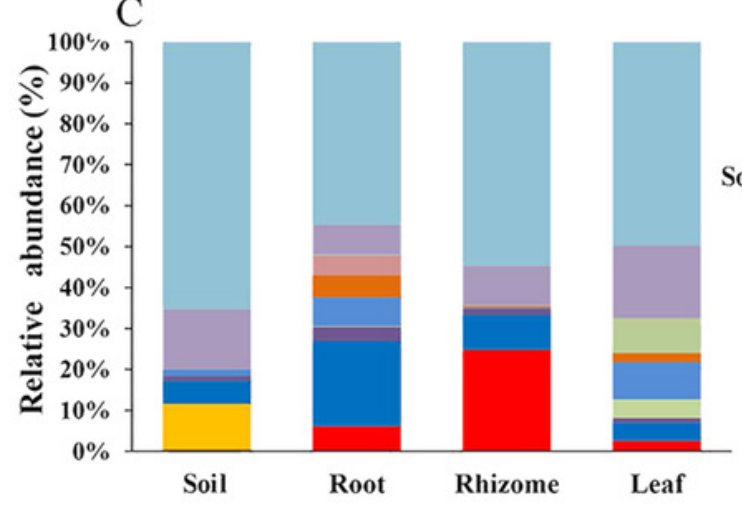

B
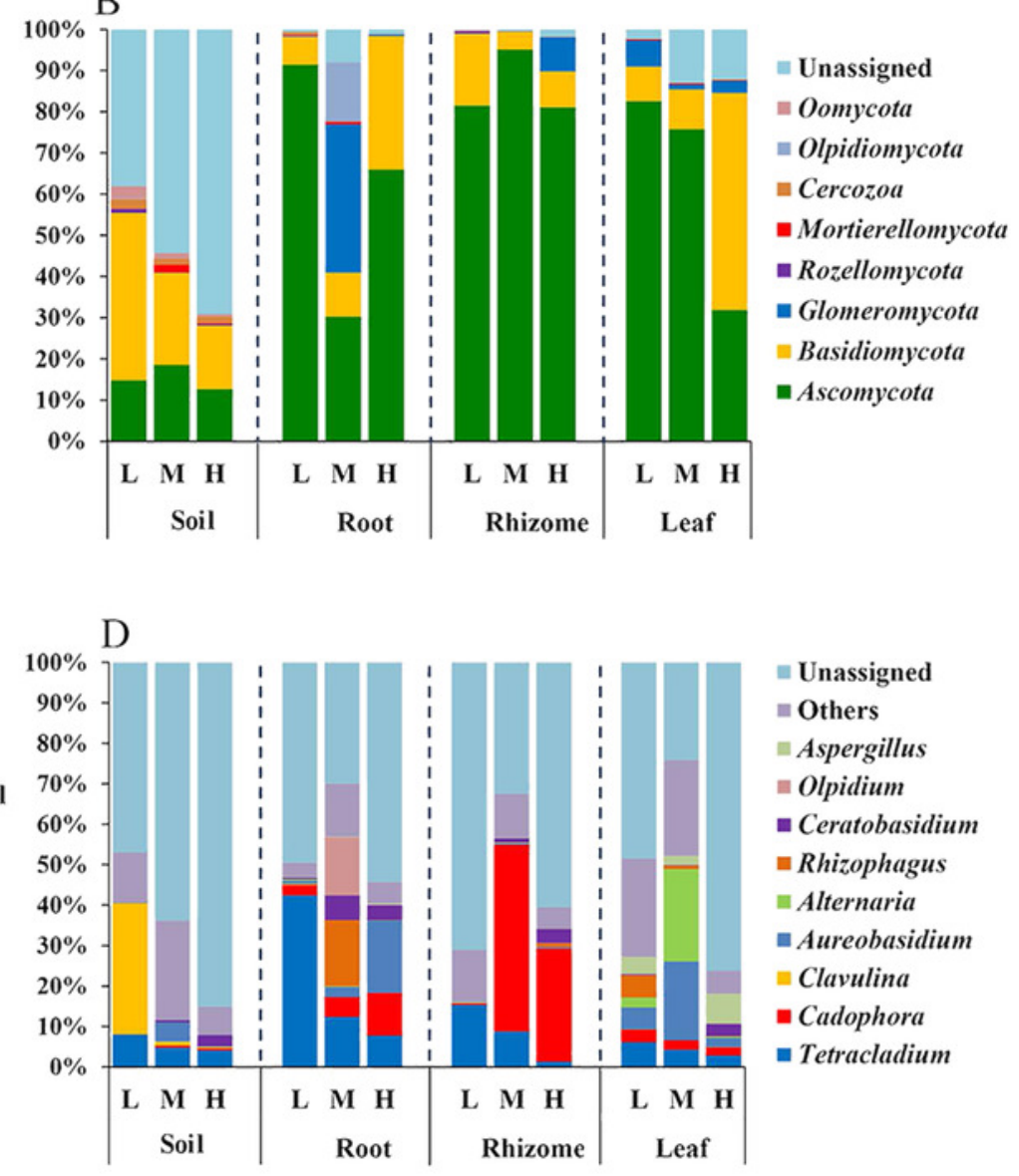


\section{Figure 6}

Venn diagrams of the number of OTUs in different mycobiota .

The numbers within overlapping regions of ellipses are the total numbers of OTUs shared among the samples, and the numbers of unique OTUs in each sample are presented in the non-overlapping regions. The proportion of exclusive OTU in the soil was $22.89 \%$, and the proportion of OTU in the plants was $34.15 \%$. The OTUs simultaneously detected in soil and plants was $42.96 \%$.

\section{Rhizome}

\section{Root}

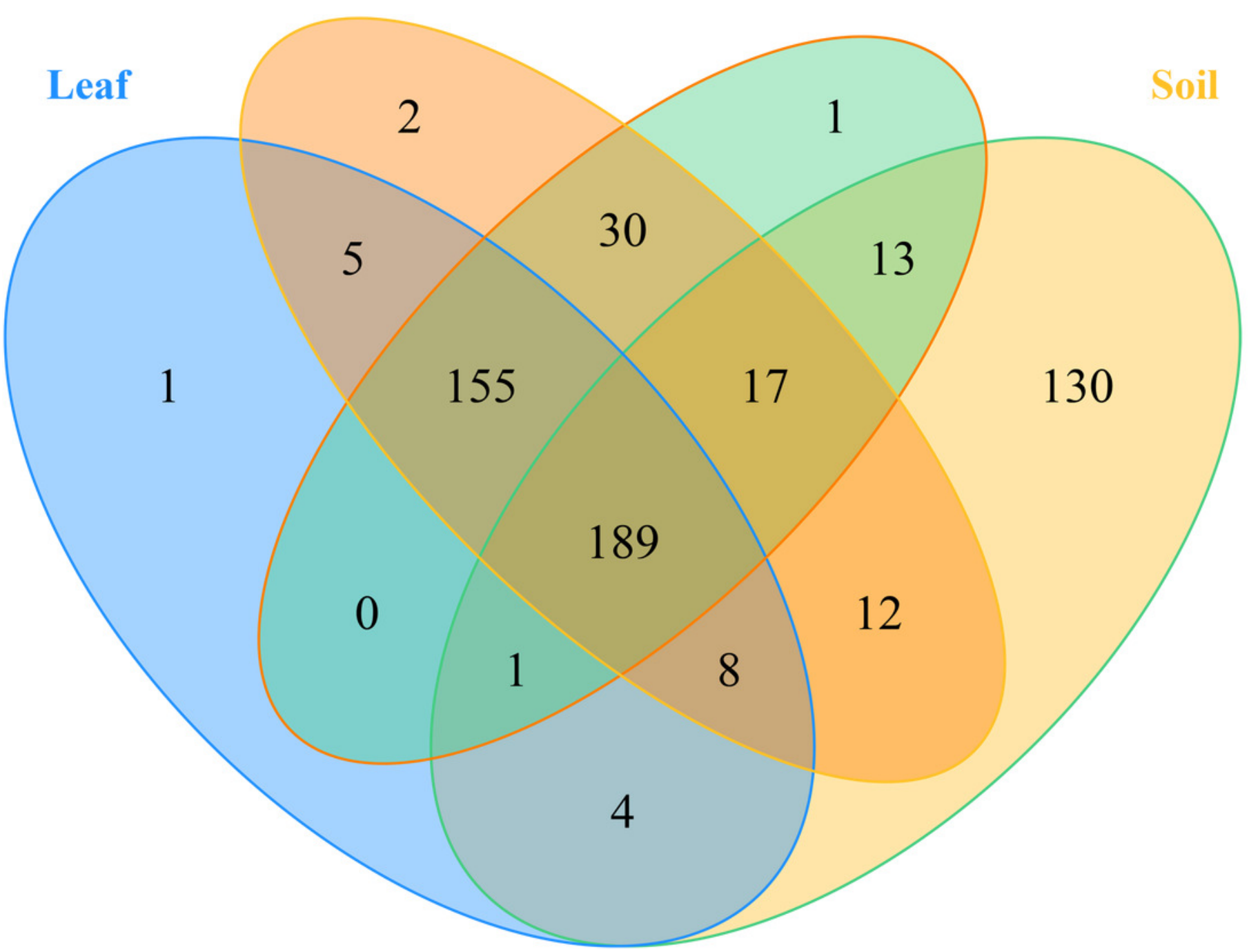




\section{Figure 7}

Cluster heat maps of phylum and genus abundances .

A: The abundances of different phyla at each elevation are shown. The horizontal cluster is the sample information at different altitudes; the longitudinal cluster is the species information; the left cluster tree is the species clustering tree; the right side contains the information of the species belonging to the phylum. B: Heat map representing the core microbiome at the genus level. The different colours indicate the prevalence of the corresponding genera: red, high prevalence; blue, low prevalence. Cadophora was the core mycobiota type in the rhizome.

A

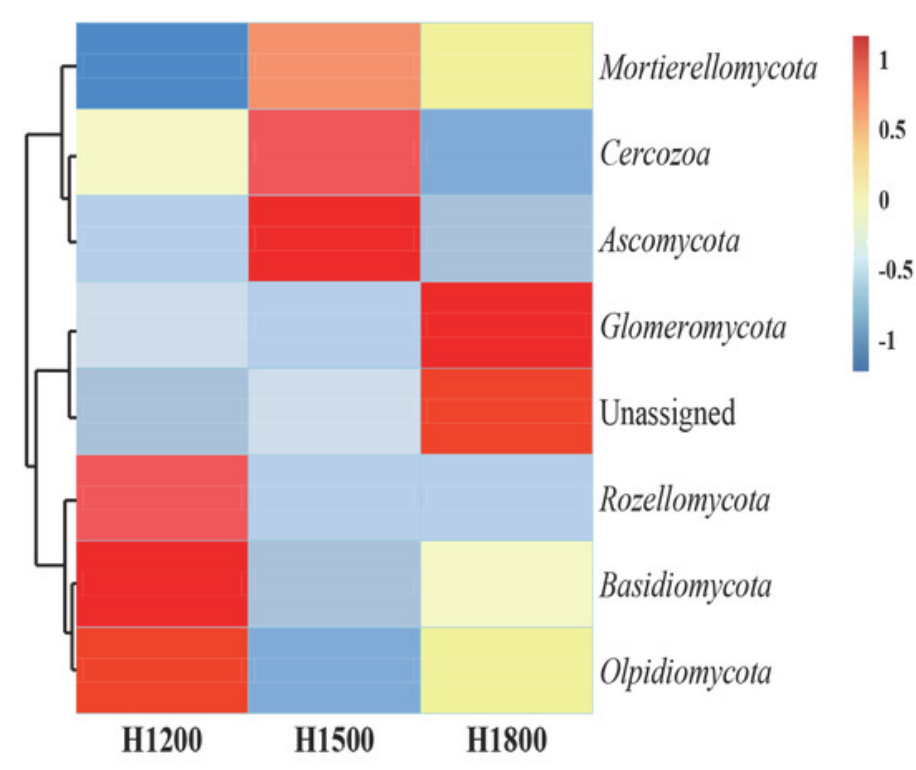

$\mathrm{B}$

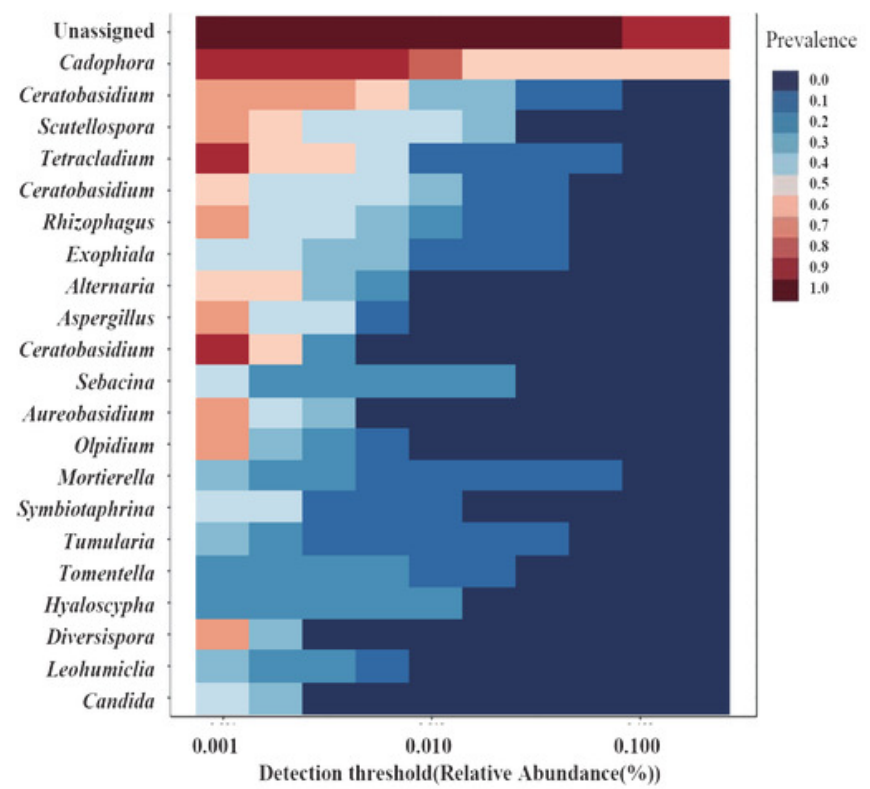


Figure 8

Phylogenetic tree

The same colour name represents the same phylum. The phylogenetic tree was constructed using the Python tool. Each branch in the tree represents a species, and the length of the branch is the evolutionary distance between two species, namely, the degree of difference between the species. 


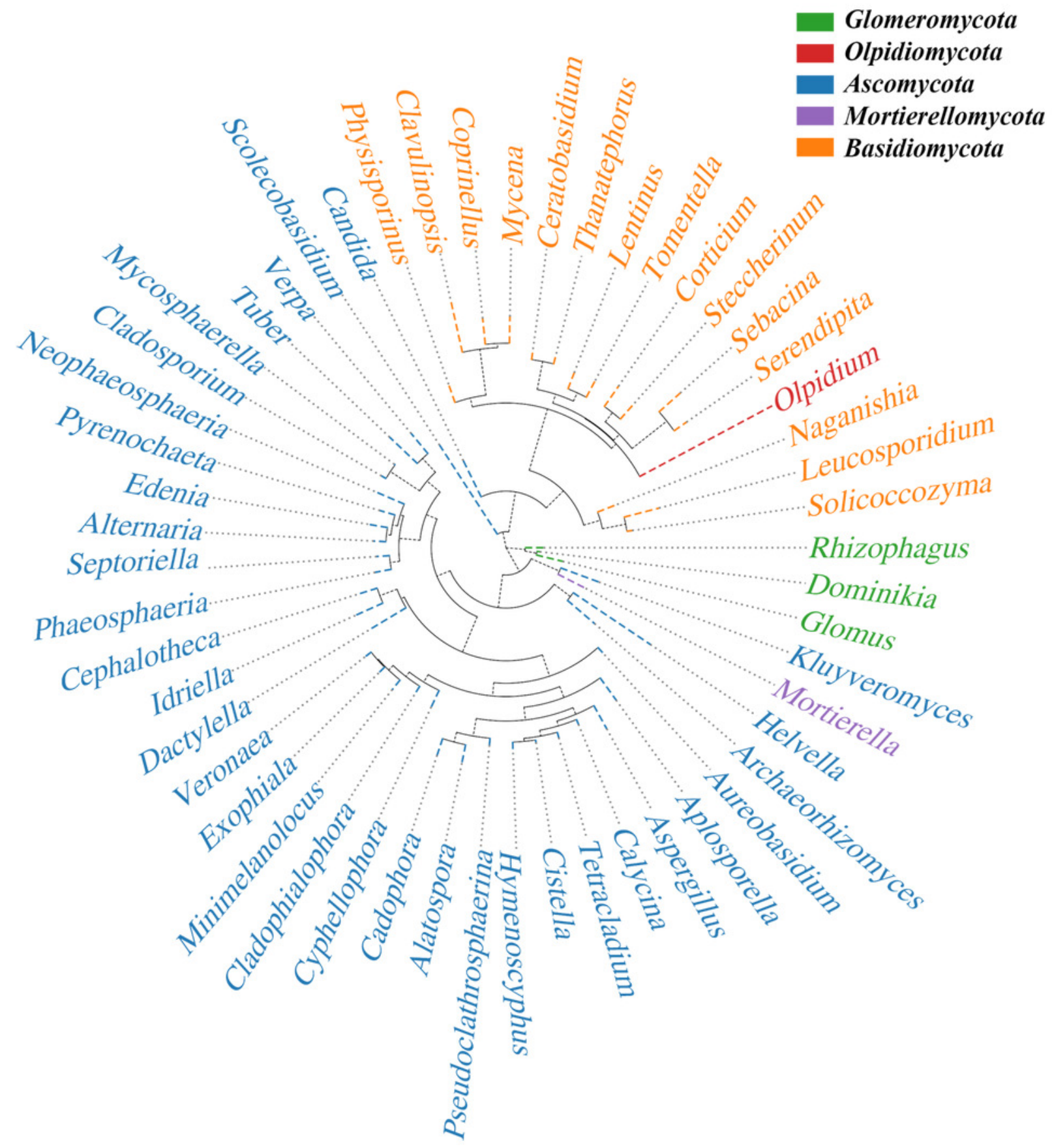




\section{Table $\mathbf{1}$ (on next page)}

Quality metrics of pyrosequencing analysis

Quality metrics of pyrosequencing analysis A: Quality metrics before and after quality control(QC); the average read length was calculated based on 36 samples across all plant compartments. B: Average number of assigned reads per plant compartment and undefined percentage of classified reads ( \pm standard deviation). Each plant compartment was evaluated separately, and the data are from all samples in each mycobiota. 
1 Table 1

2 Quality metrics of pyrosequencing analysis

\begin{tabular}{|c|c|c|c|c|}
\hline \multicolumn{5}{|c|}{ A. Total number of reads and read length before and after quality checking and trimming } \\
\hline & $\begin{array}{l}\text { Total \# of raw } \\
\text { reads before QC }\end{array}$ & $\begin{array}{l}\text { Average read length } \\
\text { before QC }\end{array}$ & $\begin{array}{l}\text { Total \# of assigned } \\
\text { reads after QC }\end{array}$ & $\begin{array}{l}\text { Average read } \\
\text { length after QC }\end{array}$ \\
\hline & 6159204 & $301 \mathrm{bp}$ & 4369448 & $222 \mathrm{bp} \pm 21$ \\
\hline \multicolumn{5}{|l|}{ B. Base and reads } \\
\hline & Soil & Root & Rhizome & Leaf \\
\hline Combined_base (bp) & 160245381 & 303613851 & 409652197 & 115621552 \\
\hline Combined_reads & 665185 & 1437855 & 1712012 & 554396 \\
\hline $\begin{array}{l}\text { Average \# of } \\
\text { Combined reads }\end{array}$ & 73909 & 159762 & 190224 & 61600 \\
\hline Uncombined (\%) & $46.16 \pm 20$ & $16.68 \pm 9$ & $22.74 \pm 30$ & $28.23 \pm 12$ \\
\hline
\end{tabular}

3 Quality metrics of pyrosequencing analysis A: Quality metrics before and after quality control (QC); the average read length was

4 calculated based on 36 samples across all plant compartments. B: Average number of assigned reads per plant compartment and

5 undefined percentage of classified reads ( \pm standard deviation). Each plant compartment was evaluated separately, and the data

6 are from all samples in each mycobiota.

7 


\section{Table 2 (on next page)}

Analysis of similarity.

Plant compartment effects on the fungal community structures were calculated using ANOSIM (analysis of similarities) with the Spearman rank correl ation method Plant compartments (soil, root, stem, leaf) were a priori defined groups at OTU phylogenetic levels. Significance levels: ${ }^{* P} \leq 0.01 ;{ }^{* * P} \leq 0.001 ;{ }^{* * * P} \leq 0.0001$. ANOSIM test statistic. 
1 Table 2

2 Analysis of similarity (ANOSIM)

\begin{tabular}{lll}
\hline Phylogenetic level ANOSIM output & $\mathrm{R}$ & $\mathrm{P}$ \\
\hline Soil VS Root & 0.997 & $0.001^{* *}$ \\
Soil VS Rhizome & 0.940 & $0.001^{* *}$ \\
Soil VS Leaf & 0.987 & $0.001^{* *}$ \\
Root VS Rhizome VS Leaf & 0.06 & 0.915 \\
\hline
\end{tabular}

3 Plant compartment effects on the fungal community structures were calculated using ANOSIM (analysis of similarities) with the

4 Spearman rank correlation method Plant compartments (soil, root, stem, leaf) were a priori defined groups at OTU phylogenetic 5 levels. Significance levels: $* \mathrm{P} \leq 0.01 ; * * \mathrm{P} \leq 0.001 ;{ }^{* * *} \mathrm{P} \leq 0.0001$. ANOSIM test statistic. 